\title{
Multi-operator colligations and multivariate characteristic functions
}

\author{
Yury A. Neretin 1
}

In the spectral theory of non-self-adjoint operators there is a well-known operation of product of operator colligations. Many similar operations appear in the theory of infinite-dimensional groups as multiplications of double cosets. We construct characteristic functions for such double cosets and get semigroups of matrix-valued functions in matrix balls.

\section{Introduction}

1.1. Operator colligations. Originally, operator colligations and characteristic functions appeared in the spectral theory of non-selfadjoint operators in 1946-55 in works of M. S. Livshits and V. P. Potapov (see, 8, [9], 18, for expositions, see [5], 1]). We discuss definitions in a minimal generality and do not touch spectral theory and related function theory (see [14).

We say that a unitary operator $U$ in a Hilbert space is finite if rank of $U-1$ is finite.

Consider a finite dimensional Euclidean space $\mathcal{H}$, and an infinite-dimensional Hilbert space $\mathcal{K} \simeq \ell_{2}$. An operator colligation is a finite unitary operator

$$
\mathfrak{A}=\left(\begin{array}{ll}
A & B \\
C & D
\end{array}\right): \mathcal{H} \oplus \mathcal{K} \rightarrow \mathcal{H} \oplus \mathcal{K}
$$

determined up to a conjugation

$$
\left(\begin{array}{ll}
A & B \\
C & D
\end{array}\right) \sim\left(\begin{array}{cc}
1 & 0 \\
0 & U
\end{array}\right)\left(\begin{array}{ll}
A & B \\
C & D
\end{array}\right)\left(\begin{array}{cc}
1 & 0 \\
0 & U^{-1}
\end{array}\right)
$$

where $U: \mathcal{K} \rightarrow \mathcal{K}$ is a unitary operator.

A product of operator colligations

$$
\mathfrak{A}=\left(\begin{array}{cc}
A & B \\
C & D
\end{array}\right), \quad \mathfrak{P}=\left(\begin{array}{ll}
P & Q \\
R & T
\end{array}\right)
$$

is given by the formula

$$
\left(\begin{array}{cc}
A & B \\
C & D
\end{array}\right) \circ\left(\begin{array}{ll}
P & Q \\
R & T
\end{array}\right):=\left(\begin{array}{lll}
A & B & 0 \\
C & D & 0 \\
0 & 0 & 1
\end{array}\right)\left(\begin{array}{ccc}
P & 0 & Q \\
0 & 1 & 0 \\
R & 0 & T
\end{array}\right)=\left(\begin{array}{ccc}
A P & B & A Q \\
C P & D & C Q \\
R & 0 & T
\end{array}\right) .
$$

We get an operator $\mathcal{H} \oplus \mathcal{K} \oplus \mathcal{K} \rightarrow \mathcal{H} \oplus \mathcal{K} \oplus \mathcal{K}$. But all infinite-dimensional separable Hilbert spaces are isomorphic, we identify $\mathcal{K} \oplus \mathcal{K} \simeq \mathcal{K}$ in arbitrary way and come to an operator $\mathcal{H} \oplus \mathcal{K} \rightarrow \mathcal{H} \oplus \mathcal{K}$.

\footnotetext{
${ }^{1}$ Supported by grants FWF, P22122 and P19064.
} 
Theorem 1.1 a) The multiplication $\circ$ is well-defined on the set of operator colligations.

b) The operation $\circ$ is associative.

Verification is straightforward.

1.2. Characteristic functions. Denote by $\overline{\mathbb{C}}=\mathbb{C} \cup \infty$ the Riemann sphere. We define a characteristic function of an operator colligation $\mathfrak{A}=\left(\begin{array}{ll}A & B \\ C & D\end{array}\right)$ by

$$
\chi(\mathfrak{A} ; z)=A+z B(1-z D)^{-1} C, \quad z \in \overline{\mathbb{C}} .
$$

Theorem 1.2 a) $\chi(\mathfrak{A} ; z)$ is a rational matrix-valued function.

b) $\chi(\mathfrak{A} \circ \mathfrak{P} ; z)=\chi(\mathfrak{A}) \chi(\mathfrak{P} ; z)$

Theorem 1.3 a) $\|\chi(\mathfrak{A} ; z)\| \leqslant 1$ for $|z|<1$.

b) $\chi(\mathfrak{A} ; z)$ is unitary for $|z|=1$.

c) $\chi\left(\mathfrak{A}, \bar{z}^{-1}\right)=\chi(\mathfrak{A} ; z)^{*-1}$.

Generally speaking, an operator colligation can not be uniquely reconstructed from its characteristic function. The reason is the following. The operator colligations

$$
\mathfrak{A}:=\left(\begin{array}{cc}
A & B \\
C & D
\end{array}\right), \quad \mathfrak{A}:=\left(\begin{array}{ccc}
A & B^{\prime} & 0 \\
C^{\prime} & D^{\prime} & 0 \\
0 & 0 & L
\end{array}\right), \quad \text { where } L \text { is unitary }
$$

have the same characteristic function.

For a colligation $\mathfrak{A}:=\left(\begin{array}{ll}A & B \\ C & D\end{array}\right)$ we denote by $\Xi(\mathfrak{A})$ the set of eigenvalues of $D$ lying on the unit circle taking in account multiplicities (the multiplicity of $z=1$ is $\infty)$.

REMARK. The spectrum of the block $D$ is contained in the circle $|\lambda| \leqslant 1$. It can be shown that for $|\lambda|<1$, the point $z=\lambda^{-1}$ is a pole of the characteristic function.

Theorem 1.4 Any operator colligation can be uniquely reconstructed from $\chi(\mathfrak{A} ; z)$ and $\Xi(\mathfrak{A})$.

Proposition $1.5 \Xi(\mathfrak{A} \circ \mathfrak{P})$ is $\Xi(\mathfrak{A}) \cup \Xi(\mathfrak{P})$ taking in account multiplicities.

Denote by $G / / L$ conjugacy classes of a group $G$ with respect to a subgroup $L$. We can regard $\circ$ as an operations on conjugacy classes

$$
\mathrm{U}_{\alpha+n} / / \mathrm{U}_{n} \times \mathrm{U}_{\alpha+m} / / \mathrm{U}_{m} \rightarrow \mathrm{U}_{\alpha+n+m} / / \mathrm{U}_{n+m}
$$

We also can reject the unitarity condition. Then we come to a multiplication

$$
\mathrm{GL}_{\alpha+n} / / \mathrm{GL}_{n} \times \mathrm{GL}_{\alpha+m} / / \mathrm{GL}_{m} \rightarrow \mathrm{GL}_{\alpha+n+m} / / \mathrm{GL}_{n+m}
$$


Then Theorems 1.1, 1.2 survive, Theorem 1.3 disappear. Theorem 1.4 exists in a weaker form (see, e.g., 4]).

In fact there is lot of operations of this type. An independent origin is explained in the following two subsections.

Below we prefer to discuss unitary groups and not GL.

1.3. Another stand-point. Infinite-dimensional classical groups, see [16], 10. Consider some series $G(n) / K(n)$ of Riemannian symmetric spaces, say $\mathrm{U}(n) / \mathrm{O}(n)$. Here $\mathrm{U}(n)$ is the unitary group and $\mathrm{O}(n)$ is the real orthogonal group. Consider double coset:2

$$
K(n-\alpha) \backslash G(n) / K(n-\alpha)=\mathrm{O}(n-\alpha) \backslash \mathrm{U}(n) / \mathrm{O}(n-\alpha) .
$$

They form a 'hypergroup' in the following sense. Let $g \in \mathrm{U}(n)$. Denote by $\mu_{g}$ the natural probability measure on the set $K(n-\alpha) g K(n-\alpha)$. Consider the convolution of measures

$$
\mu_{g_{1}} * \mu_{g_{2}}=\int \mu_{h} d \lambda(h)
$$

where $\lambda(h)$ is a probability measure on $K(n-\alpha) \backslash G / K(n-\alpha)$. Thus we get a map

$$
\begin{aligned}
& K(n-\alpha) \backslash G(n) / K(n-\alpha) \times K(n-\alpha) \backslash G(n) / K(n-\alpha) \rightarrow \\
& \rightarrow\{\text { probability measures on } K(n-\alpha) \backslash G(n) / K(n-\alpha)\}
\end{aligned}
$$

Explicit description of this hypergroup even for $\alpha=0$ is complicated 3 .

However, if we pass to a limit as $n \rightarrow \infty$ (and keep $\alpha$ fixed), then the measure $\nu$ is concentrated near a single double coset 4 and a well-defined operation

$$
\begin{aligned}
K(\infty-\alpha) \backslash G(\infty) / K(\infty-\alpha) \times & K(\infty-\alpha) \backslash G(\infty) / K(\infty-\alpha) \rightarrow \\
& \rightarrow K(\infty-\alpha) \backslash G(\infty) / K(\infty-\alpha)
\end{aligned}
$$

Note, that the last operation is not a convolution of measures (because there is no a natural measure on $K(\infty-\alpha) g K(\infty-\alpha))$.

For instance, consider the case $\mathrm{O}(\infty-\alpha) \backslash \mathrm{U}(\infty) / \mathrm{O}(\infty-\alpha)$, i.e. finite $(\alpha+\infty) \times(\alpha+\infty)$ unitary matrices defined up to the equivalence

$$
\left(\begin{array}{ll}
A & B \\
C & D
\end{array}\right) \sim\left(\begin{array}{cc}
1 & 0 \\
0 & U
\end{array}\right)\left(\begin{array}{ll}
A & B \\
C & D
\end{array}\right)\left(\begin{array}{cc}
1 & 0 \\
0 & V
\end{array}\right)
$$

where $U, V$ are orthogonal matrices. The multiplication of double cosets is given by the formula (1.3).

\footnotetext{
${ }^{2}$ Let $G$ be a group, $K$ a subgroup. A double coset is a set of the form $K g K \subset G$. The notation for sets of all double cosets is $K \backslash G / K$.

${ }^{3}$ As far as I know formulas for $K(n) \backslash G(n) / K(n)$ exist only for rank 1 groups and for complex groups. See formulas for $\mathrm{O}(2) \backslash \mathrm{SL}(2, \mathbb{R}) / \mathrm{O}(2)$ in $[7$, for $\mathrm{U}(n) \backslash \mathrm{GL}(n, \mathbb{C}) / \mathrm{U}(n)$ in $[2$.

${ }^{4}$ Apparently, the phenomenon of concentration was firstly observed in [15].
} 
Theorem 1.6 (Multiplicativity theorem) Let $\rho$ be a unitary irreducible representation of $\mathrm{U}(\infty)$ in a Hilbert space $H$. Denote by $H(\alpha)$ the space of $\mathrm{O}(\infty-\alpha)$ fixed vectors in $H$. Denote by $P(\alpha)$ the projection to $H(\alpha)$. Assume that at least one subspace $H(\beta)$ is non-zero. For $g \in \mathrm{U}(n)$ define the operator

$$
\bar{\rho}_{\alpha}(g)=P(\alpha) \rho(g): H(\alpha) \rightarrow H(\alpha) .
$$

Then $\bar{\rho}_{\alpha}(g)$ is a function of a double coset $\mathfrak{A} \ni g$ and

$$
\bar{\rho}_{\alpha}\left(\mathfrak{A}_{1}\right) \bar{\rho}_{\alpha}\left(\mathfrak{A}_{2}\right)=\bar{\rho}_{\alpha}\left(\mathfrak{A}_{1} \circ \mathfrak{A}_{2}\right)
$$

Note that neither the product (1.6), nor multiplicativity theorem (1.7) have finite-dimensional analogs.

A description of this multiplication in the terms of characteristic functions and some additional data is given in [10], IX.4. Olshansky paper [16] provides us a zoo of such constructions related to infinite-dimensional symmetric spaces.

1.4. Purposes of the paper. In [10] it was observed that multiplications of double cosets and multiplicativity theorems exist under rather weak restrictions. Let $G$ be an infinite-dimensional classical group, $K$ be its subgroup isomorphic to a complete unitary group $\mathrm{U}(\infty)$ (or $\mathrm{O}(\infty), \mathrm{Sp}(\infty)$ ), the subgroup is equipped with a weak operator topology. Then usually there is a multiplication

$$
K \backslash G / K \times K \backslash G / K \rightarrow K \backslash G / K
$$

and usually the multiplicativity theorem holds.

This produces numerous operations of the type (1.3). Oue purpose is to transfer such operations to multiplications of meromorphic multi-variate matrixvalued functions. We explain the technology in Sections 2-3, it can be applied in numerous situations. Some further examples are discussed Sections 3-5.

Note that nontrivial representation-theoretical constructions related to infinitedimensional non-symmetric pairs $G \supset K$ were considered in [12, [13] and [11].

\subsection{Notation. Below}

- Mat $(n)$ is the space of $n \times n$ matrices;

- $A^{t}, A^{*}$ are transposed matrix and adjoint matrix; on $\mathbb{C}^{k}$,

$-\langle\cdot, \cdot\rangle,(\cdot, \cdot)$ are the standard inner product and the standard bilinear form

$$
\langle p, q\rangle=\sum_{j=1}^{k} p_{j} \bar{q}_{j} ; \quad(p, q)=\sum_{j=1}^{k} p_{j} q_{j} ;
$$

- $\mathrm{U}(n), \mathrm{U}(p, q), \operatorname{Sp}(2 n, \mathbb{C})$, are the usual notation for classical groups; $\mathrm{U}(\infty)$ denotes the group of finite unitary matrices.

Acknowledgements. I am grateful to S. L. Tregub and A .A. Rosly for discussion of this topic. 


\section{Multiple colligations}

2.1. Multiple colligations. We say that an $n$-colligation is a collection $\mathfrak{A}$ of unitary $(\alpha+\infty) \times(\alpha+\infty)$-matrices $g_{j}=\left(\begin{array}{ll}a_{j} & b_{j} \\ c_{j} & d_{j}\end{array}\right)$, where $j=1, \ldots, n$, defined up to simultaneous conjugation

$$
\begin{aligned}
& \left\{\left(\begin{array}{ll}
a_{1} & b_{1} \\
c_{1} & d_{1}
\end{array}\right), \ldots,\left(\begin{array}{ll}
a_{n} & b_{n} \\
c_{n} & d_{n}
\end{array}\right)\right\} \sim \\
& \sim\left\{\left(\begin{array}{ll}
1 & 0 \\
0 & u
\end{array}\right)\left(\begin{array}{ll}
a_{1} & b_{1} \\
c_{1} & d_{1}
\end{array}\right)\left(\begin{array}{ll}
1 & 0 \\
0 & u
\end{array}\right)^{-1}, \ldots,\left(\begin{array}{ll}
1 & 0 \\
0 & u
\end{array}\right)\left(\begin{array}{ll}
a_{n} & b_{n} \\
c_{n} & d_{n}
\end{array}\right)\left(\begin{array}{ll}
1 & 0 \\
0 & u
\end{array}\right)^{-1}\right\},
\end{aligned}
$$

where $u$ is unitary.

2.2. Product of multiple colligations is determined element-wise,

$$
\left\{\left(\begin{array}{cc}
a_{j} & b_{j} \\
c_{j} & d_{j}
\end{array}\right)\right\} \circ\left\{\left(\begin{array}{cc}
\widetilde{a}_{j} & \widetilde{b}_{j} \\
\widetilde{c}_{j} & \widetilde{d}_{j}
\end{array}\right)\right\}=\left\{\left(\begin{array}{cc}
a_{j} & b_{j} \\
c_{j} & d_{j}
\end{array}\right) \circ\left(\begin{array}{ll}
\widetilde{a}_{j} & \widetilde{b}_{j} \\
\widetilde{c}_{j} & \widetilde{d}_{j}
\end{array}\right)\right\} .
$$

2.3. Characteristic functions. For definiteness, set $n=3$. Fix a $3 \times 3$-matrix $S=\left\{s_{i j}\right\}$. We write the equation

$$
\left(\begin{array}{c}
q_{1} \\
s_{11} x_{1}+s_{12} x_{2}+s_{13} x_{3} \\
q_{2} \\
s_{21} x_{1}+s_{22} x_{2}+s_{23} x_{3} \\
q_{3} \\
s_{31} x_{1}+s_{32} x_{2}+s_{33} x_{3}
\end{array}\right)=\left(\begin{array}{cccccc}
a_{1} & b_{1} & 0 & 0 & 0 & 0 \\
c_{1} & d_{1} & 0 & 0 & 0 & 0 \\
0 & 0 & a_{2} & b_{2} & 0 & 0 \\
0 & 0 & c_{2} & d_{2} & 0 & 0 \\
0 & 0 & 0 & 0 & a_{3} & b_{3} \\
0 & 0 & 0 & 0 & c_{3} & d_{3}
\end{array}\right)\left(\begin{array}{c}
p_{1} \\
x_{1} \\
p_{2} \\
x_{2} \\
p_{2} \\
x_{3}
\end{array}\right)
$$

or

$$
\begin{aligned}
q_{1} & =a_{1} p_{1}+b_{1} x_{1} \\
s_{11} x_{1}+s_{12} x_{2}+s_{13} x_{3} & =c_{1} p_{1}+d_{1} x_{1} \\
q_{2} & =a_{2} p_{2}+b_{2} x_{2} \\
s_{21} x_{1}+s_{22} x_{2}+s_{23} x_{3} & =c_{2} p_{2}+d_{2} x_{2} \\
q_{3} & =a_{3} p_{3}+b_{3} x_{3} \\
s_{31} x_{1}+s_{32} x_{2}+s_{33} x_{3} & =c_{3} p_{3}+d_{3} x_{3}
\end{aligned}
$$

Next, we exclude 'indeterminantes' $x_{1}, x_{2}, x_{3}$ from (2.3), (2.5), (2.7). Substituting $x_{1}, x_{2}, x_{3}$ to (2.2), (2.4), (2.6) we get a certain dependence of the form

$$
\left(\begin{array}{l}
q_{1} \\
q_{2} \\
q_{3}
\end{array}\right)=\chi(\mathfrak{A} ; S)\left(\begin{array}{l}
p_{1} \\
p_{2} \\
p_{3}
\end{array}\right),
$$

where $\chi(\mathfrak{A} ; S) \in \operatorname{Mat}(n \alpha)$ (above $n=3$ ). 
Consider the eigensurface $\Xi[\mathfrak{A}]$ of $d_{1}, d_{2}, d_{3}$ in the space of $3 \times 3$ matrices determined by the equation

$$
\operatorname{det}\left(\begin{array}{ccc}
s_{11}-d_{1} & s_{12} & s_{13} \\
s_{21} & s_{22}-d_{2} & s_{23} \\
s_{31} & s_{32} & s_{33}-d_{3}
\end{array}\right)=0 .
$$

(here $d_{1}, \ldots, d_{3}$ are given and $s_{i j}$ are indeterminances.5. If $S \notin \Xi(\mathfrak{A})$, then the equations (2.3), (2.5), (2.7) have a unique solution.

Now let $n$ arbitrary.

Theorem 2.1 a) $\chi(\mathfrak{A} ; S)$ is a meromorphic matrix-valued function on the space of $n \times n$-matrices, whose singularities are contained in the eigensurface $\Xi[\mathfrak{A}]$.

b) $\chi(\mathfrak{A} ; S)$ depends only on the operator colligations but not on matrices $\left(\begin{array}{ll}a_{j} & b_{j} \\ c_{j} & d_{j}\end{array}\right)$ themselves.

c) The following identity holds

$$
\chi(\mathfrak{A} ; S) \chi(\mathfrak{P} ; S)=\chi(\mathfrak{A} \circ \mathfrak{P} ; S)
$$

pointwise.

d) If $\|S\|<1$, then $\chi(\mathfrak{A} ; S)$ is expanding, i.e., it satisfies

$$
\left\|\chi(\mathfrak{A} ; S)^{-1}\right\|<1
$$

If $\|S\|=1$, then we have $\left\|\chi(\mathfrak{A} ; S)^{-1}\right\|=1$.

e) In particular, the matrix valued function $\chi(\mathfrak{A} ; S)^{-1}$ is holomorphic in the matrix ball $\|S\|<1$.

f) If $S$ is unitary, then $\chi(\mathfrak{A} ; S)$ is unitary.

g) The following Riemann-Schwarz type identity holds

$$
\chi\left(\mathfrak{A} ; S^{*-1}\right)=\chi(\mathfrak{A} ; S)^{*-1}
$$

h) Let $\lambda_{1}, \ldots, \lambda_{n} \in \mathbb{C}^{*}$. Let $\lambda$ be the diagonal matrix with entries $\lambda_{j}$, and $\Lambda$ be $(\alpha+\cdots+\alpha) \times(\alpha+\cdots+\alpha)$ block diagonal matrix with blocks $\lambda_{j}$. Then

$$
\chi\left(\mathfrak{A} ; \lambda S \lambda^{-1}\right)=\Lambda \chi(\mathfrak{A} ; S) \Lambda^{-1} .
$$

2.4. Proof of Theorem [2.1, a If $S$ is not on the eigensurface, then the equations (2.3), (2.5), (2.7) have a unique solution $x_{1}, x_{2}, x_{3}$ for given $p_{1}, p_{2}$, $p_{3}$. Therefore the equations (2.2), (2.4), (2.6) uniquely determine $q_{1}, q_{2}, q_{3}$ from $p_{1}, p_{2}, p_{3}$.

\footnotetext{
${ }^{5}$ There are two points of view to spectral data of several matrices $A_{j}$. The first one is related to determinantal hypersurfaces, see a survey of Beauville [3. The second is related to spectral curves, which are widely explored in the theory of integrable systems, see an introduction of Hitchin [6]. It seems that characteristic functions give the third point of view.
} 
2.5. Proof of Theorem 2.1.b. To be definite, set $n=2$. Consider a multiple colligation equivalent to a given one. We write the equation

$$
\left(\begin{array}{c}
q_{1} \\
s_{11} x_{1}+s_{12} x_{2} \\
q_{2} \\
s_{21} x_{1}+s_{22} x_{2}
\end{array}\right)=\left(\begin{array}{cccc}
1 & 0 & 0 & 0 \\
0 & u^{-1} & 0 & 0 \\
0 & 0 & 1 & 0 \\
0 & 0 & 0 & u^{-1}
\end{array}\right)\left(\begin{array}{cccc}
a_{1} & b_{1} & 0 & 0 \\
c_{1} & d_{1} & 0 & 0 \\
0 & 0 & a_{2} & b_{2} \\
0 & 0 & c_{2} & d_{2}
\end{array}\right)\left(\begin{array}{cccc}
1 & 0 & 0 & 0 \\
0 & u & 0 & 0 \\
0 & 0 & 1 & 0 \\
0 & 0 & 0 & u
\end{array}\right)\left(\begin{array}{l}
p_{1} \\
x_{1} \\
p_{2} \\
x_{2}
\end{array}\right)
$$

or

$$
\left(\begin{array}{c}
q_{1} \\
s_{11} u x_{1}+s_{12} u x_{2} \\
q_{2} \\
s_{21} u x_{1}+s_{22} u x_{2}
\end{array}\right)=\left(\begin{array}{cccc}
a_{1} & b_{1} & 0 & 0 \\
c_{1} & d_{1} & 0 & 0 \\
0 & 0 & a_{2} & b_{2} \\
0 & 0 & c_{2} & d_{2}
\end{array}\right)\left(\begin{array}{c}
p_{1} \\
u x_{1} \\
p_{2} \\
u x_{2}
\end{array}\right)
$$

We denote

$$
y_{1}=u x_{1} \quad y_{2}=u x_{2}
$$

and come to the system determining $\chi(\mathfrak{A} ; S)$.

2.6. Proof of Theorem 2.1.c. To be definite (and to have finite size of matrices), take $n=2$. We have

$$
\begin{aligned}
& \left(\begin{array}{c}
q_{1} \\
s_{11} x_{1}+s_{12} x_{2} \\
q_{2} \\
s_{21} x_{1}+s_{22} x_{2}
\end{array}\right)=\left(\begin{array}{cccc}
a_{1} & b_{1} & 0 & 0 \\
c_{1} & d_{1} & 0 & 0 \\
0 & 0 & a_{2} & b_{2} \\
0 & 0 & c_{2} & d_{2}
\end{array}\right)\left(\begin{array}{l}
p_{1} \\
x_{1} \\
p_{2} \\
x_{2}
\end{array}\right) \\
& \left(\begin{array}{c}
p_{1} \\
s_{11} y_{1}+s_{12} y_{2} \\
p_{2} \\
s_{21} y_{1}+s_{22} x y_{2}
\end{array}\right)=\left(\begin{array}{cccc}
\widetilde{a}_{1} & \widetilde{b}_{1} & 0 & 0 \\
\widetilde{c}_{1} & \widetilde{d}_{1} & 0 & 0 \\
0 & 0 & \widetilde{a}_{2} & \widetilde{b}_{2} \\
0 & 0 & \widetilde{c}_{2} & \widetilde{d}_{2}
\end{array}\right)\left(\begin{array}{l}
r_{1} \\
y_{1} \\
r_{2} \\
y_{2}
\end{array}\right)
\end{aligned}
$$

Then

$$
\begin{gathered}
\left(\begin{array}{c}
q_{1} \\
s_{11} x_{1}+s_{12} x_{2} \\
s_{11} y_{1}+s_{12} y_{2} \\
q_{2} \\
s_{21} x_{1}+s_{22} x_{2} \\
s_{21} y_{1}+s_{22} y_{2}
\end{array}\right)=\left(\begin{array}{cccccc}
a_{1} & b_{1} & 0 & 0 & 0 & 0 \\
c_{1} & d_{1} & 0 & 0 & 0 & 0 \\
0 & 0 & 1 & 0 & 0 & 0 \\
0 & 0 & 0 & a_{2} & b_{2} & 0 \\
0 & 0 & 0 & c_{2} & d_{2} & 0 \\
0 & 0 & 0 & 0 & 0 & 1
\end{array}\right)\left(\begin{array}{c}
p_{1} \\
x_{1} \\
s_{11} y_{1}+s_{12} y_{2} \\
p_{2} \\
x_{2} \\
s_{21} y_{1}+s_{22} y_{2}
\end{array}\right)= \\
=\left(\begin{array}{cccccc}
a_{1} & b_{1} & 0 & 0 & 0 & 0 \\
c_{1} & d_{1} & 0 & 0 & 0 & 0 \\
0 & 0 & 1 & 0 & 0 & 0 \\
0 & 0 & 0 & a_{2} & b_{2} & 0 \\
0 & 0 & 0 & c_{2} & d_{2} & 0 \\
0 & 0 & 0 & 0 & 0 & 1
\end{array}\right)\left(\begin{array}{cccccc}
\widetilde{a}_{1} & 0 & \widetilde{b}_{1} & 0 & 0 & 0 \\
0 & 1 & 0 & 0 & 0 & 0 \\
\widetilde{c}_{1} & 0 & \widetilde{d}_{1} & 0 & 0 & 0 \\
0 & 0 & 0 & \widetilde{a}_{2} & 0 & \widetilde{b}_{2} \\
0 & 0 & 0 & 0 & 1 & 0 \\
0 & 0 & 0 & \widetilde{c}_{2} & 0 & \widetilde{d}_{2}
\end{array}\right)\left(\begin{array}{c}
r_{1} \\
x_{1} \\
y_{1} \\
r_{2} \\
x_{2} \\
y_{2}
\end{array}\right)
\end{gathered}
$$

We get a product of colligations in the right-hand side. 
SS

2.7. Proof of Theorem 2.1,d-f. Let us prove d). Since the big matrix in (2.1) is unitary, we have

$\left\|q_{1}\right\|^{2}+\left\|s_{11} x_{1}+s_{12} x_{2}\right\|^{2}+\left\|q_{2}\right\|^{2}+\left\|s_{21} x_{1}+s_{22} x_{2}\right\|^{2}=\left\|p_{1}\right\|^{2}+\left\|x_{1}\right\|^{2}+\left\|p_{2}\right\|^{2}+\left\|x_{2}\right\|^{2}$

Since $\|S\| \leqslant 1$, we have

$$
\left\|s_{11} x_{1}+s_{12} x_{2}\right\|^{2}+\left\|s_{21} x_{1}+s_{22} x_{2}\right\|^{2} \leqslant\left\|x_{1}\right\|^{2}+\left\|x_{2}\right\|^{2}
$$

and therefore

$$
\left\|q_{1}\right\|^{2}+\left\|q_{2}\right\|^{2} \geqslant\left\|p_{1}\right\|^{2}+\left\|p_{2}\right\|^{2}
$$

If $S$ is unitary, then we have $=$ in (2.9) and therefore $=$ in (2.10).

2.8. Proof of Theorem 2.1.g. Let $n=2$. Since matrices $\left(\begin{array}{ll}a_{1} & b_{1} \\ c_{1} & d_{1}\end{array}\right)$, $\left(\begin{array}{ll}a_{2} & b_{2} \\ c_{2} & d_{2}\end{array}\right)$ are unitary, we can write (2.1) as

$$
\left(\begin{array}{c}
q_{1} \\
s_{11} x_{1}+s_{12} x_{2} \\
q_{2} \\
s_{21} x_{1}+s_{22} x_{2}
\end{array}\right)=\left(\begin{array}{cc}
\left(\begin{array}{cc}
a_{1}^{*} & c_{1}^{*} \\
b_{1}^{*} & d_{1}^{*} \\
0 & 0 \\
0 & 0
\end{array} \quad\left(\begin{array}{cc}
0 & 0 \\
0 & 0 \\
a_{2}^{*} & c_{2}^{*} \\
b_{2}^{*} & d_{2}^{*}
\end{array}\right)^{-1}\right.
\end{array}\right)\left(\begin{array}{l}
p_{1} \\
x_{1} \\
p_{2} \\
x_{2}
\end{array}\right)
$$

or

$$
\left(\begin{array}{l}
p_{1} \\
x_{1} \\
p_{2} \\
x_{2}
\end{array}\right)=\left(\begin{array}{cccc}
a_{1}^{*} & c_{1}^{*} & 0 & 0 \\
b_{1}^{*} & d_{1}^{*} & 0 & 0 \\
0 & 0 & a_{2}^{*} & c_{2}^{*} \\
0 & 0 & b_{2}^{*} & d_{2}^{*}
\end{array}\right)\left(\begin{array}{c}
q_{1} \\
s_{11} x_{1}+s_{12} x_{2} \\
q_{2} \\
s_{121} x_{1}+s_{22} x_{2}
\end{array}\right)
$$

Now we change variables

$$
\left\{\begin{array} { l } 
{ y _ { 1 } = s _ { 1 1 } x _ { 1 } + s _ { 1 2 } x _ { 2 } } \\
{ y _ { 2 } = s _ { 2 1 } x _ { 1 } + s _ { 2 2 } x _ { 2 } }
\end{array} \quad \left\{\begin{array}{l}
x_{1}=\sigma_{11} y_{1}+\sigma_{12} y_{2} \\
x_{2}=\sigma_{21} y_{1}+\sigma_{22} y_{2}
\end{array}\right.\right.
$$

and come to

$$
\left(\begin{array}{c}
p_{1} \\
\sigma_{11} y_{1}+s_{12} y_{2} \\
p_{2} \\
\sigma_{21} y_{1}+\sigma_{22} y_{2}
\end{array}\right)=\left(\begin{array}{cccc}
a_{1}^{*} & c_{1}^{*} & 0 & 0 \\
b_{1}^{*} & d_{1}^{*} & 0 & 0 \\
0 & 0 & a_{2}^{*} & c_{2}^{*} \\
0 & 0 & b_{2}^{*} & d_{2}^{*}
\end{array}\right)\left(\begin{array}{c}
q_{1} \\
y_{1} \\
q_{2} \\
y_{2}
\end{array}\right)
$$

2.9. Proof of Theorem 2.1,h. Set $n=2$. We write (2.1) as

$$
\begin{aligned}
& \left(\begin{array}{c}
q_{1} \\
s_{11} x_{1}+s_{12} x_{2} \\
q_{2} \\
s_{21} x_{1}+s_{22} x_{2}
\end{array}\right)= \\
& =\left(\begin{array}{cccc}
\lambda_{1} & 0 & 0 & 0 \\
0 & \lambda_{1} & 0 & 0 \\
0 & 0 & \lambda_{2} & 0 \\
0 & 0 & 0 & \lambda_{2}
\end{array}\right) \quad\left(\begin{array}{cccc}
a_{1} & b_{1} & 0 & 0 \\
c_{1} & d_{1} & 0 & 0 \\
0 & 0 & a_{2} & b_{2} \\
0 & 0 & c_{2} & d_{2}
\end{array}\right)\left(\begin{array}{cccc}
\lambda_{1} & 0 & 0 & 0 \\
0 & \lambda_{1} & 0 & 0 \\
0 & 0 & \lambda_{2} & 0 \\
0 & 0 & 0 & \lambda_{2}
\end{array}\right)\left(\begin{array}{l}
p_{1} \\
x_{1} \\
p_{2} \\
x_{2}
\end{array}\right)
\end{aligned}
$$


We denote

$$
y_{1}=\lambda_{1} x_{1}, \quad y_{2}=\lambda_{2} x_{2},
$$

and write

$$
\left(\begin{array}{c}
\lambda_{1} p_{1} \\
s_{11} y_{1}+s_{12} \lambda_{1}^{-1} \lambda_{2} y_{2} \\
\lambda_{2} p_{2} \\
s_{21} \lambda_{1} \lambda_{2}^{-1} y_{1}+s_{22} y_{2}
\end{array}\right)=\left(\begin{array}{cccc}
a_{1} & b_{1} & 0 & 0 \\
c_{1} & d_{1} & 0 & 0 \\
0 & 0 & a_{2} & b_{2} \\
0 & 0 & c_{2} & d_{2}
\end{array}\right)\left(\begin{array}{c}
\lambda_{1} p_{1} \\
y_{1} \\
\lambda_{2} p_{2} \\
y_{2}
\end{array}\right)
$$

\section{$3 \quad$ Language of Grassmannians}

Here the rephrase the construction of the previous section.

3.1. Linear relations. Let $V, W$ be linear spaces. A linear relation $P: V \rightrightarrows W$ is a subspace in $V \oplus W$. The product $Q R$ of linear relations $P: V \rightrightarrows W, Q: W \rightrightarrows Y$ is a linear relation consisting of $v \oplus y \in V \oplus Y$ such that there exists $w \in W$ satisfying $v \oplus w \in P, w \oplus y \in Q$.

Example. Let $A: V \rightarrow W$ is linear operator. Then the graph $\operatorname{graph}(A)$ of $A$ is a linear relation.

3.2. Eigensurface. Denote by $\mathrm{Gr}_{p, q}$ the Grassmannian of $p$-dimensional subspaces in $\mathbb{C}^{p+q}$. Let us keep the notation of the previous section.

Consider the space $\mathbb{C}^{n} \oplus \mathbb{C}^{n}$ with coordinates $\left(v_{1}, \ldots, v_{n}, w_{1}, \ldots, w_{n}\right)$.

Fix an $n$-dimensional subspace $L \subset \mathbb{C}^{n} \oplus \mathbb{C}^{n}$. Let

$$
\sum s_{i j} v_{j}+\sum \sigma_{i j} w_{j}=0
$$

be a collection of equations determining $L$.

For definiteness, set $n=3$. We write the equation

$$
\left(\begin{array}{c}
0 \\
y_{1} \\
0 \\
y_{2} \\
0 \\
y_{3}
\end{array}\right)=\left(\begin{array}{cccccc}
a_{1} & b_{1} & 0 & 0 & 0 & 0 \\
c_{1} & d_{1} & 0 & 0 & 0 & 0 \\
0 & 0 & a_{2} & b_{2} & 0 & 0 \\
0 & 0 & c_{2} & d_{2} & 0 & 0 \\
0 & 0 & 0 & 0 & a_{3} & b_{3} \\
0 & 0 & 0 & 0 & c_{3} & d_{3}
\end{array}\right)\left(\begin{array}{c}
0 \\
x_{1} \\
0 \\
x_{2} \\
0 \\
x_{3}
\end{array}\right)
$$

or, equivalently,

$$
\left(\begin{array}{l}
y_{1} \\
y_{2} \\
y_{3}
\end{array}\right)=\left(\begin{array}{ccc}
d_{1} & 0 & 0 \\
0 & d_{2} & 0 \\
0 & 0 & d_{3}
\end{array}\right)\left(\begin{array}{l}
x_{1} \\
x_{2} \\
x_{3}
\end{array}\right)
$$

We say that $L \in \mathrm{Gr}_{n, n}$ is contained in the eigensurface $\Xi[\mathfrak{A}]$ if there exists a non-zero vector $\left(x_{1}, x_{2}, x_{3}, y_{1}, y_{2}, y_{3}\right)$ satisfying (3.2) and the system

$$
\left\{\sum s_{i j} x_{j}+\sum \sigma_{i j} y_{j}=0\right.
$$


and (3.1).

3.3. Characteristic function. For definiteness, let $n=3$. Now we write the equation

$$
\left(\begin{array}{l}
q_{1} \\
y_{1} \\
q_{2} \\
y_{2} \\
q_{3} \\
y_{3}
\end{array}\right)=\left(\begin{array}{cccccc}
a_{1} & b_{1} & 0 & 0 & 0 & 0 \\
c_{1} & d_{1} & 0 & 0 & 0 & 0 \\
0 & 0 & a_{2} & b_{2} & 0 & 0 \\
0 & 0 & c_{2} & d_{2} & 0 & 0 \\
0 & 0 & 0 & 0 & a_{3} & b_{3} \\
0 & 0 & 0 & 0 & c_{3} & d_{3}
\end{array}\right)\left(\begin{array}{l}
p_{1} \\
x_{1} \\
p_{2} \\
x_{2} \\
p_{3} \\
x_{3}
\end{array}\right)
$$

For any $L \in \mathrm{Gr}_{3,3}$ we construct a linear relation

$$
X(\mathfrak{A} ; L)=\mathbb{C}^{\alpha} \oplus \mathbb{C}^{\alpha} \oplus \mathbb{C}^{\alpha} \rightrightarrows \mathbb{C}^{\alpha} \oplus \mathbb{C}^{\alpha} \oplus \mathbb{C}^{\alpha}
$$

by the following rule: $\left(p_{1}, p_{2}, p_{3} ; q_{1}, q_{2}, q_{3}\right) \in X(\mathfrak{A} ; L)$ if there exist $\left(x_{1}, x_{2}, x_{3}, y_{1}, y_{2}, y\right) 3$ satisfying (3.4) and (3.3).

Note that $X(\mathfrak{A} ; L)$ is well-defined also for $L$ being in the eigensurface. If

\section{Theorem 3.1}

$$
X(\mathfrak{A} \circ \mathfrak{P} ; L) \supset X(\mathfrak{A} ; L) \circ X(\mathfrak{P} ; L) .
$$

Proof is the same as in 2.6

3.4. Rephrasing of the expansion property. We define an indefinite Hermitian form in $\left(\mathbb{C}^{\alpha}\right)^{n} \oplus\left(\mathbb{C}^{\alpha}\right)^{n}$ by

$$
\mathcal{M}\left(p \oplus q ; p^{\prime} \oplus q^{\prime}\right)=\sum_{i=1}^{n}\left\langle p_{i}, p_{i}^{\prime}\right\rangle-\sum_{i=1}^{n}\left\langle q_{i}, q_{i}^{\prime}\right\rangle
$$

and a Hermitian form $M$ on $\mathbb{C}^{n} \oplus \mathbb{C}^{n}$ given by

$$
M\left(v \oplus w ; v^{\prime} \oplus w^{\prime}\right)=\sum_{i=1}^{n} v_{i} \bar{v}_{i}^{\prime}-\sum_{i=1}^{n} w_{i} \bar{w}_{i}^{\prime} .
$$

Theorem 3.2 If $M$ is positive definite on $L$, then $\mathcal{M}$ is negative definite on $X(\mathfrak{A} ; L)$.

\section{Conjugacy classes: another example}

4.1. Conjugacy classes. Now we consider elements of the group $\mathrm{U}(\alpha+p+p)$ up to the equivalence

$$
\left(\begin{array}{ccc}
a & b_{1} & b_{2} \\
c_{1} & d_{11} & d_{12} \\
c_{2} & d_{21} & d_{22}
\end{array}\right) \sim\left(\begin{array}{ccc}
1 & 0 & 0 \\
0 & u & 0 \\
0 & 0 & u
\end{array}\right)\left(\begin{array}{ccc}
a & b_{1} & b_{2} \\
c_{1} & d_{11} & d_{12} \\
c_{2} & d_{21} & d_{22}
\end{array}\right)\left(\begin{array}{ccc}
1 & 0 & 0 \\
0 & u & 0 \\
0 & 0 & u
\end{array}\right)^{-1}
$$


where $u \in \mathrm{U}(p)$. We denote this set by $\mathrm{U}(\alpha+2 p) / / \mathrm{U}(p)$. We have a well-defined operation

$$
\mathrm{U}(\alpha+2 p) \times \mathrm{U}(\alpha+2 q) \rightarrow \mathrm{U}(\alpha+2 p+2 q)
$$

determined by

$$
\begin{aligned}
\left(\begin{array}{ccc}
a & b_{1} & b_{2} \\
c_{1} & d_{11} & d_{12} \\
c_{2} & d_{21} & d_{22}
\end{array}\right) \circ\left(\begin{array}{ccc}
\widetilde{a} & \widetilde{b}_{1} & \widetilde{b}_{2} \\
\widetilde{c}_{1} & \widetilde{d}_{11} & \widetilde{d}_{12} \\
\widetilde{c}_{2} & \widetilde{d}_{21} & \widetilde{d}_{22}
\end{array}\right):= \\
:=\left(\begin{array}{ccccc}
a & b_{1} & 0 & b_{2} & 0 \\
c_{1} & d_{11} & 0 & d_{12} & 0 \\
0 & 0 & 1 & 0 & 0 \\
c_{2} & d_{21} & 0 & d_{22} & 0 \\
0 & 0 & 0 & 0 & 1
\end{array}\right)\left(\begin{array}{ccccc}
\widetilde{a} & 0 & \widetilde{b}_{1} & 0 & \widetilde{b}_{2} \\
0 & 1 & 0 & 0 & 0 \\
\widetilde{c}_{1} & 0 & \widetilde{d}_{11} & 0 & \widetilde{d}_{12} \\
0 & 0 & 0 & 1 & 0 \\
\widetilde{c}_{2} & 0 & \widetilde{d}_{21} & 0 & \widetilde{d}_{22}
\end{array}\right)
\end{aligned}
$$

We define the characteristic function on the space of $2 \times 2$ matrices. We write the equation

$$
\left(\begin{array}{c}
q \\
s_{11} x_{1}+s_{12} x_{2} \\
s_{21} x_{1}+s_{22} x_{2}
\end{array}\right)=\left(\begin{array}{ccc}
a & b_{1} & b_{2} \\
c_{1} & d_{11} & d_{12} \\
c_{2} & d_{21} & d_{22}
\end{array}\right)\left(\begin{array}{c}
p \\
x_{1} \\
x_{2}
\end{array}\right)
$$

Then we exclude $x_{1}, x_{2}$ and get a matrix-valued function on Mat(2).

Theorem 4.1 All the claims of Theorem 2.1 hold except $h$ ).

Proofs are the same as above.

\section{Example: double cosets}

5.1. Double cosets $\mathrm{U}(\infty) \times \cdots \times \mathrm{U}(\infty)$ with respect to $\mathrm{O}(\infty-\alpha)$. Now we consider collections of $n$ finite unitary matrices

$$
\left\{\left(\begin{array}{ll}
a_{j} & b_{j} \\
c_{j} & d_{j}
\end{array}\right)\right\}
$$

of the size $(\alpha+\infty) \times(\alpha+\infty)$ determined up to the equivalence

$$
\begin{aligned}
& \left\{\left(\begin{array}{ll}
a_{1} & b_{1} \\
c_{1} & d_{1}
\end{array}\right), \ldots,\left(\begin{array}{ll}
a_{n} & b_{n} \\
c_{n} & d_{n}
\end{array}\right)\right\} \sim \\
& \sim\left\{\left(\begin{array}{ll}
1 & 0 \\
0 & u
\end{array}\right)\left(\begin{array}{ll}
a_{1} & b_{1} \\
c_{1} & d_{1}
\end{array}\right)\left(\begin{array}{ll}
1 & 0 \\
0 & v
\end{array}\right), \ldots,\left(\begin{array}{ll}
1 & 0 \\
0 & u
\end{array}\right)\left(\begin{array}{ll}
a_{n} & b_{n} \\
c_{n} & d_{n}
\end{array}\right)\left(\begin{array}{ll}
1 & 0 \\
0 & v
\end{array}\right)\right\}
\end{aligned}
$$

where $u, v$ are finite real orthogonal matrices, $u=u^{t-1}, v=v^{t-1}$. The multiplication of colligations is defined as above. 
5.2. Characteristic functions. For definiteness, set $n=2$. We write the equation

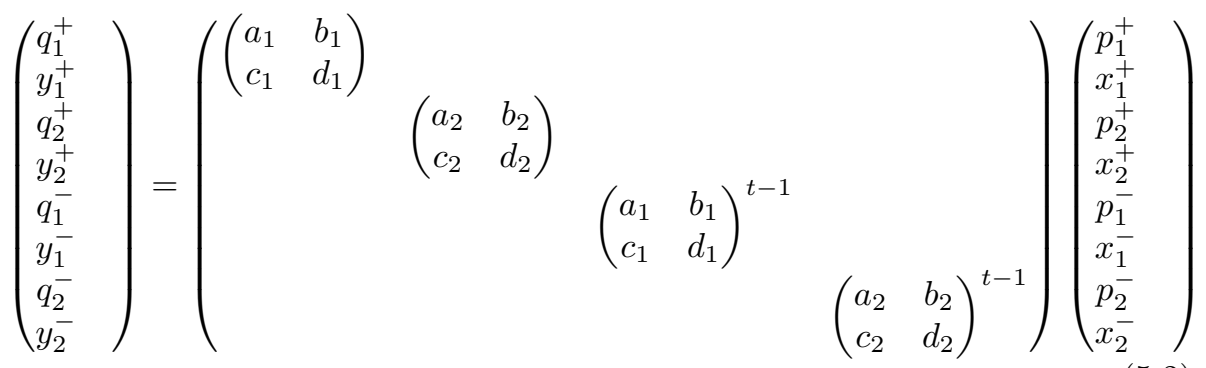

Next, take two $n \times n$ matrices $S, R$ (in our case $n=2$ ) and assume that $x$, $y$ satisfy the equations

$$
\left\{\begin{array} { l } 
{ y _ { 1 } ^ { + } = s _ { 1 1 } y _ { 1 } ^ { - } + s _ { 1 2 } y _ { 2 } ^ { - } } \\
{ y _ { 2 } ^ { + } = s _ { 2 1 } y _ { 1 } ^ { - } + s _ { 2 2 } y _ { 2 } ^ { - } }
\end{array} \quad \left\{\begin{array}{l}
x_{1}^{-}=r_{11} x_{1}^{+}+r_{12} x_{2}^{+} \\
x_{2}^{-}=r_{21} x_{1}^{+}+r_{22} x_{2}^{+}
\end{array}\right.\right.
$$

Now we exclude variables $y_{1}^{+}, y_{2}^{+}, x_{1}^{-}, x_{2}^{-}$and come to a dependence of the form

$$
\left(\begin{array}{l}
q_{1}^{+} \\
q_{2}^{+} \\
q_{1}^{-} \\
q_{2}^{-}
\end{array}\right)=\chi(\mathfrak{A} ; S, R)\left(\begin{array}{c}
p_{1}^{+} \\
p_{2}^{+} \\
p_{1}^{-} \\
p_{2}^{-}
\end{array}\right),
$$

where $\chi(\mathfrak{A} ; T, R)$ is a function of two variables $T, R \in \operatorname{Mat}(n)$ taking values in $\operatorname{Mat}(2 n \alpha)$.

Theorem 5.1 a) $\chi(\mathfrak{A} ; S, R)$ is a meromorphic matrix-valued function.

b) $\chi(\mathfrak{A} ; S, R)$ depends only of the equivalence class (5.1) and not on matrices themselves.

c) $\chi(\mathfrak{A} \circ \mathfrak{P} ; S, R)=\chi(\mathfrak{A} ; S, R) \chi(\mathfrak{A} ; S, R)$

Proofs are similar to proofs of corresponding statements of Theorem 2.1]

Theorem 2.1 also can be extended in a straightforward way. For definiteness, set $n=2$.

Theorem 5.2 Let $\lambda_{1}, \lambda_{2} \in \mathbb{C}^{*}$.

$$
\begin{gathered}
\left(\begin{array}{cccc}
\lambda_{1} & 0 & 0 & 0 \\
0 & \lambda_{2} & 0 & 0 \\
0 & 0 & \lambda_{1}^{-1} & 0 \\
0 & 0 & 0 & \lambda_{2}^{-1}
\end{array}\right) \chi(\mathfrak{A} ; S, R)\left(\begin{array}{cccc}
\lambda_{1} & 0 & 0 & 0 \\
0 & \lambda_{2} & 0 & 0 \\
0 & 0 & \lambda_{1}^{-1} & 0 \\
0 & 0 & 0 & \lambda_{2}^{-1}
\end{array}\right)^{-1}= \\
=\chi\left[\mathfrak{A} ;\left(\begin{array}{cc}
\lambda_{1} & 0 \\
0 & \lambda_{2}
\end{array}\right) S\left(\begin{array}{cc}
\lambda_{1} & 0 \\
0 & \lambda_{2}
\end{array}\right)^{-1},\left(\begin{array}{cc}
\lambda_{1} & 0 \\
0 & \lambda_{2}
\end{array}\right) R\left(\begin{array}{cc}
\lambda_{1} & 0 \\
0 & \lambda_{2}
\end{array}\right)^{-1}\right]
\end{gathered}
$$


5.3. The analog of expansion property. We define an Hermitian form $\mathcal{M}$ in the space $V:=\left(\mathbb{C}^{\alpha}\right)^{n} \oplus\left(\mathbb{C}^{\alpha}\right)^{n}$ by

$\mathcal{M}\left(p_{1}^{+}, \ldots, p_{n}^{+}, p_{1}^{-}, \ldots, p_{n}^{-} ; \widetilde{p}_{1}^{+}, \ldots, \widetilde{p}_{n}^{+}, \widetilde{p}_{1}^{-}, \ldots, \widetilde{p}_{n}^{-}\right)=\sum_{j=1}^{n}\left\langle p_{j}^{+}, \widetilde{p}_{j}^{+}\right\rangle-\sum_{j=1}^{n}\left\langle p_{j}^{-}, \widetilde{p}_{j}^{-}\right\rangle$.

Theorem 5.3 a) Let $\|S\|<1,\|R\|<1$. Let $q=\chi(\mathfrak{A} ; S, R) p$. Then

$$
M(q, q)>M(p, p) .
$$

b) Let $S, R$ be unitary, let $(S, R)$ be a point of holomorpy of $\chi(\mathfrak{A} ; S, R)$. Then $\chi(\mathfrak{A} ; S, R)$ is contained in the pseudo-unitary group $\mathrm{U}(n \alpha, n \alpha)$, i.e.,

$$
M(q, q)=M(p, p) \quad \text { if } q=\chi(\mathfrak{A} ; S, R) p .
$$

c) The following Riemann-Scwartz type identity holds

$$
\chi\left(\mathfrak{A} ; S^{\square-1}, R^{\square-1}\right)=\chi(\mathfrak{A} ; S, R)^{\square-1}
$$

where $\square$ denotes the adjoint operator with respect to the form $M$.

Proof. We prove a). We introduce the following indefinite Hermitian form $\mathbf{M}$ on the space $V \oplus \ell_{2}$,

$\mathbf{M}\left(p^{+}, p^{-}, x^{+}, x^{-} ; \widetilde{p}^{+}, \widetilde{p}^{-}, \widetilde{x}^{+}, \widetilde{x}^{-}\right)=\left\langle p^{+}, \widetilde{p}^{+}\right\rangle+\left\langle x^{+}, \widetilde{x}^{+}\right\rangle-\left\langle p^{-}, \widetilde{p}^{-}\right\rangle-\left\langle x^{-}, \widetilde{x}^{-}\right\rangle$.

The big matrix in (5.2) preserves this form. Therefore, for $(p, x)$ and $(q, y)$ related by (5.2) the following identity holds

$$
\left\|p^{+}\right\|^{2}-\left\|p^{-}\right\|^{2}+\left\|x^{+}\right\|^{2}-\left\|x^{-}\right\|^{2}=\left\|q^{+}\right\|^{2}-\left\|q^{-}\right\|^{2}+\left\|y^{+}\right\|^{2}-\left\|y^{-}\right\|^{2} .
$$

Now we note that $x_{-}=R x_{+}, y+=S y_{-}$. Therefore

$$
\left\|x^{-}\right\|^{2}<\left\|x^{+}\right\|^{2} \quad\left\|y^{+}\right\|^{2}<\left\|y^{-}\right\|^{2}
$$

This implies the required statement.

REMARK. In Theorem 2.1] $\chi^{-1}$ is holomorphic in the matrix ball, this is not valid here.

5.4. An additional symmetry. In our case, there is an additional property of characteristic functions comparing(?) Theorem 2.1.

Let us introduce the following skew-symmetric bilinear form $\Lambda$ in $V$,

$$
\Lambda(p, \widetilde{p})=\sum_{j=1}^{n}\left(\left(p_{j}^{+}, \widetilde{p}_{j}^{-}\right)-\left(p_{j}^{-} \widetilde{p}_{j}^{+}\right)\right) . .
$$

Denote by ${ }^{\triangle}$ the transposition with respect to the form $\Lambda$,

$$
\Lambda(g p, \widetilde{p})=\Lambda\left(p, g^{\triangle} \widetilde{p}\right) .
$$


Theorem 5.4 a)

$$
\chi\left(\mathfrak{A} ; S^{t}, R^{t}\right)=\chi(\mathfrak{A} ; S, R)^{\triangle-1} .
$$

b) In particular, if matrices $S, R$ are symmetric, then $\chi(\mathfrak{A} ; T, S)$ is contained in the symplectic group $\operatorname{Sp}(2 n \alpha, \mathbb{C})$.

Proof. We introduce a skew-symmetric bilinear form $\mathcal{L}$ in $V \oplus \ell_{2}$ by

$$
\mathcal{L}(p \oplus x, \widetilde{p} \oplus \widetilde{x})=\sum_{j=1}^{n}\left(\left(p_{j}^{+}, \widetilde{p}_{j}^{-}\right)-\left(p_{j}^{-}, \widetilde{p}_{j}^{+}\right)\right)+\sum_{j=1}^{n}\left(\left(x_{j}^{+}, \widetilde{x}_{j}^{-}\right)-\left(x_{j}^{-}, \widetilde{x}_{j}^{+}\right)\right) .
$$

Let $p \oplus x, q \oplus y$ (and also $\widetilde{p} \oplus \widetilde{x}, \widetilde{q} \oplus \widetilde{y}$ ) satisfy (5.2). Let $x, y$ be connected by (5.3) and $\widetilde{x}, \widetilde{y}$ be connected by the same relation (5.3), where $R, S$ are replaced by $R^{t}, S^{t}$. Then

$$
\mathcal{L}(p \oplus x, \widetilde{p} \oplus \widetilde{x})=\mathcal{L}(q \oplus y, \widetilde{q} \oplus \widetilde{y})
$$

Let

$$
y^{+}=S y^{-}, \quad \widetilde{y}^{+}=S^{t} y^{-} . \quad x^{-}=R x^{+}, \quad \widetilde{x}^{-}=R^{t} x^{+} .
$$

Then

$$
\begin{aligned}
\left(y^{+}, \widetilde{y}^{-}\right)-\left(y^{-}, \widetilde{y}^{+}\right)=\left(S y^{-}, \widetilde{y}^{-}\right)-\left(y^{-}, S^{t} \widetilde{y}^{+}\right) & =0 ; \\
\left(x^{+}, \widetilde{x}^{-}\right)-\left(x^{-}, \widetilde{x}^{+}\right)=\left(x^{+}, R \widetilde{x}^{+}\right)-\left(R^{t} x^{+}, \widetilde{x}^{+}\right) & =0 .
\end{aligned}
$$

Therefore

$$
\Lambda(q, \widetilde{q})=\Lambda(p, \widetilde{p})
$$

\section{References}

[1] Arov, D. Z., Dym, H. J-contractive matrix valued functions and related topics. Cambridge University Press, Cambridge, 2008.

[2] Berezin, F. A.; Gelfand, I. M. Some remarks on the theory of spherical functions on symmetric Riemannian manifolds. Trudy Moskov. Mat. Obs. 5 (1956), 311351; English transl. in Amer. Math. Soc. Transl. (2) 211962 193-238.

[3] Beauville, A. Determinantal hypersurfaces. Michigan Math. J. 48 (2000), 39-64.

[4] Dym, H. Linear algebra in action. American Mathematical Society, Providence, RI, 2007

[5] Gohberg, I., Goldberg, S., Kaashoek, M. A. Classes of linear operators. Vol. II. Birkhauser, Basel, 1993.

[6] Hitchin, N., Riemann surfaces and integrable systems in Hitchin, N. J.(4-OX); Segal, G. B.; Ward, R. S., Integrable systems. Twistors, loop groups, and Riemann surfaces. Oxford University Press, New York, 1999

[7] Koornwinder, T. H. Jacobi functions and analysis on noncompact semisimple Lie groups. In Special functions: group theoretical aspects and applications, 185, Math. Appl., Reidel, Dordrecht, 1984 
[8] Livshits, M.S. On a certain class of linear operators in Hilbert space. Mat. Sb., N. Ser. 19(61), 239-262 (1946); English transl. in Amer. Math. Soc. Transl. (Ser. 2), Vol. 13, 61-83 1960

[9] Livshits, M. S. On spectral decomposition of linear nonself-adjoint operators. Mat. Sbornik N.S. 34(76), (1954). 145-199. English transl. in Amer. Math. Soc. Transl. (Ser. 2), Vol 5, 1957, 67-114

[10] Neretin, Yu. A. Categories of symmetries and infinite-dimensional groups. Oxford University Press, New York, 1996; Russian transl.: URSS, 1998.

[11] Neretin, Yu. A., Infinite tri-symmetric group, multiplication of double cosets, and checker topological field theories. Preprint, available via arXiv:0909.4739

[12] Nessonov, N. I., Factor-representation of the group $G L(\infty)$ and admissible representations GL $(\infty)^{X}$, J. Math. Phys., Analysis, Geometry, 2003, N4, 167-187.

[13] Nessonov, N.I. Factor-representation of the group $G L(\infty)$ and admissible representations of $G L(\infty)^{X}$. II. Mat. Fiz. Anal. Geom. 10, No. 4, 524-556 (2003).

[14] Nikolskii, N. K. Treatise on the shift operator. Spectral function theory. SpringerVerlag, Berlin, 1986.

[15] Olshanski, G. I. New "large" groups of type I. Current problems in mathematics, Vol. 16 (Russian), pp. 31-52, 228, Akad. Nauk SSSR, Vsesoyuz. Inst. Nauchn. i Tekhn. Informatsii (VINITI), Moscow, 1980. English transl. J. Sov. Math. 18 (1982) 22-3

[16] Olshanski, G.I. Unitary representations of infinite dimensional pairs $(G, K)$ and the formalism of $R$. Howe. in Representation of Lie groups and related topics, Adv. Stud. Contemp. Math. 7, 269-463 (1990).

[17] Olshanski, G. I. Caractéres généralisés de $\mathrm{U}(\infty)$ et fonctions intérieures. [Generalized characters of $\mathrm{U}(\infty)$ and inner functions] C. R. Acad. Sci. Paris Sér. I Math. 313 (1991), no. 1, 9-12.

[18] Potapov, V. P. The multiplicative structure of J-contractive matrix functions. Trudy Moskov. Mat. Obshchestva. 4 (1955), 125-236; English transl. Amer. Math. Soc. Transl. (2) 15 (1960) 131-243

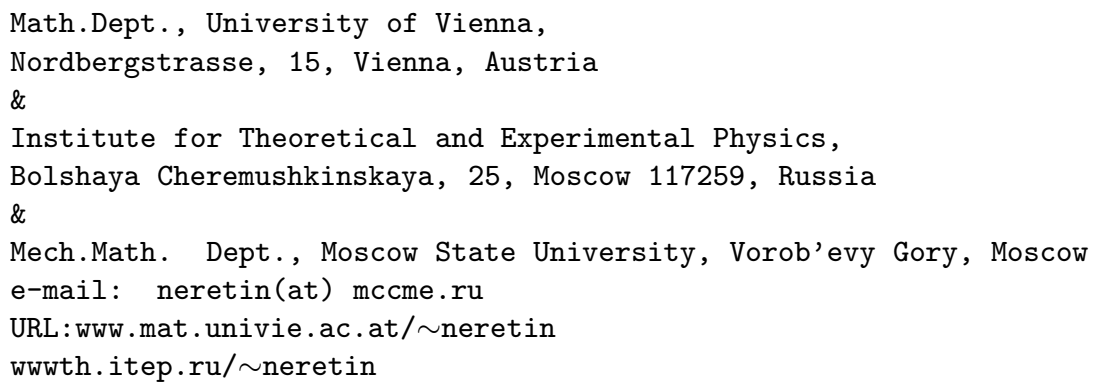

